\title{
New Approach for T-Wave Peak Detection and T-Wave End Location in 12-lead Paced ECG Signals based on a Mathematical Model
}

\author{
João P.V. Madeiro ${ }^{1,2}$, William B. Nicolson ${ }^{3}$, Paulo C. Cortez ${ }^{1}$, João A. L. Marques ${ }^{4}$, Carlos R. \\ Vázquez-Seisdedos ${ }^{5}$, Narmadha Elangovan ${ }^{2}$, G. Andre $\mathrm{Ng}^{3}$, Fernando S. Schlindwein ${ }^{2,3}$ \\ ${ }^{1}$ Department of Engineering of Teleinformatics, Federal University of Ceará, Fortaleza, Brazil \\ ${ }^{2}$ Department of Engineering, University of Leicester, Leicester, United Kingdom \\ ${ }^{3}$ Department of Cardiovascular Sciences, University of Leicester, Leicester, United Kingdom \\ ${ }^{4}$ Department of Informatics, University Lusíada of Angola, Lobito, Angola \\ ${ }^{5}$ Centre for Neurosciences Studies, Image and Signal Processing, University of Oriente, Santiago de Cuba, Cuba
}

\begin{abstract}
This paper presents an innovative approach for T-wave peak detection and subsequent $\mathbf{T}$-wave end location in 12-lead paced ECG signals based on a mathematical model of a skewed Gaussian function. Following the stage of QRS segmentation, we establish search windows using a number of the earliest intervals between each QRS offset and subsequent QRS onset. Then, we compute a template based on a Gaussian-function, modified by a mathematical procedure to insert asymmetry, which models the Twave. Cross-correlation and an approach based on the computation of Trapezium's area are used to locate, respectively, the peak and end point of each T-wave throughout the whole raw ECG signal. For evaluating purposes, we used a database of high resolution 12-lead paced ECG signals, recorded from patients with ischemic cardiomyopathy (ICM) in the University Hospitals of Leicester NHS Trust, UK, and the well-known QT database. The average $T$-wave detection rates, sensitivity and positive predictivity, were both equal to $99.12 \%$, for the first database, and, respectively, equal to $99.32 \%$ and $\mathbf{9 9 . 4 7 \%}$, for QT database. The average time errors computed for T-wave peak and T-wave end locations were, respectively, $-0.38 \pm 7.12 \mathrm{~ms}$ and $-\mathbf{3 . 7 0} \pm 15.46 \mathrm{~ms}$, for the first database, and $1.40 \pm 8.99 \mathrm{~ms}$ and $\mathbf{2 . 8 3} \pm \mathbf{1 5 . 2 7} \mathrm{ms}$, for QT database. The results demonstrate the accuracy, consistency and robustness of the proposed method for a wide variety of T-wave morphologies studied.
\end{abstract}

Index Terms - T-wave peak, T-wave end, T-wave modelling, skewed Gaussian function, cross-correlation, paced electrocardiogram (paced ECG).

\section{Introduction}

The electrocardiogram (ECG) consists of the measurement of electrical activity on the body surface associated with myocardial contraction with respect to time. Each cardiac cycle in the ECG is normally characterised by a sequence of waveforms known as $\mathrm{P}$ wave, QRS complex and T wave, so that time intervals between onset and offset of different waves are significant because they reflect physiological processes of the heart and of the autonomous nervous system [1]. Regarding paced ECG, its computerized analysis is essential to follow-up evaluation of patients with implanted pacemakers and also presents other important applications such as assessment of ventricular arrhythmia and sudden death risk in patients with ischemic cardiomyopathy [2] and prediction of reduction of left ventricular and systolic volume after cardiac resynchronization therapy [3].

Concerning T-wave, its detection and segmentation following QRS segmentation provide the beat-to-beat analysis of the time intervals between the QRS onset and the T-wave end, known as QT intervals, whose prolongation is a marker of risk for ventricular arrhythmias [4]. The QT intervals depend on the accuracy with which both points (onset and offset) are determined. A recent technique, 
microvolt T-wave alternans (MTWA) has the ability to identify patients at high risk for sudden cardiac death. Its spectral method of analysis allows detection of beat-to-beat alternans in the microvolt range of T-wave amplitudes [5,6].

Another measurement used for the duration of repolarisation is the $\mathrm{QT}_{\text {apex }}$. The beginning of the $\mathrm{QT}_{\text {apex }}$ interval is defined in the same way as that for the QT interval, but its end point is defined as the peak amplitude of the T-wave on each lead [7,8]. Because of the known difficulties in identifying $\mathrm{T}$-wave end, $\mathrm{QT}_{\text {apex }}$ has been used in medical applications where a precise ECG fiducial point has been required. In a recently published approach, Nicolson et al. have developed a novel ventricular arrhythmia risk marker, R2I2, based on the analysis of diastolic intervals and action potential durations in paced ECG signals [2]. The diastolic interval was taken as the period from T-wave peak on the last beat of a drive train to the subsequent QRS onset of an extra-stimulus, and the surrogate action potential duration was taken as the period from the QRS onset of the same extra-stimulus to the subsequent $\mathrm{T}$-wave peak.

T-wave end location is one of the most difficult ECG waveform boundary location problems, due to the slow transition of the signal around each T-wave end $[4,9,10]$. Furthermore, according to Murray et al. [10], manual measurement of QT interval exhibits considerable inter and intra-observer variability. Various automatic methods have been proposed for both detection and segmentation of Twave, based on: trapezium's area approach [4], accumulative area approach [11], mathematical models of the ECG [9,14], discrete wavelet-based estimators using the derivative of a smoothing function as the prototype wavelet [15], singular value decomposition (SVD) of multiple lead ECG signals [12], ECG curve length transform [13], phasor transformed ECG and instantaneous phase variation [17], other approaches based on Wavelet transform [18,19], pattern recognition techniques [22], among other methods.

The idea of mathematical modelling segments of the cardiac cycle is by no means new. Firstly, the purpose was to provide tools for the spectral analysis of the real cardiac signal departing from a modelled signal. Richardson et al. [23] proposed three mathematical models for individually modelling P-wave, QRS complex and T-wave using Gaussian functions and their first and second derivatives. In more recent developments, mathematical modelling is part of a dual-stage framework. A parametric function related to a segment of the cardiac cycle is obtained and algorithms for waveform characterization are applied starting with the parameters of the model fitted [24,25,26].

Padrini et al. [26] and Vila et al. [9] presented a method that uses a simple parametric function to model the Action Potential (AP). Four APs are obtained, where the differences between the first two ones will model the morphology of the T-wave, whilst the differences between the other two relate to the U-wave. After obtaining the model, they provide the detection of initial and final points of each wave (TU complex), as well as their maximum or maxima for biphasic waves, not by working on the real signal, but on the modelled one. The method is robust to noise and can adapt itself to various T-wave morphologies, but it is highly expensive in terms of computing effort, given that the process of parameterisation (made for each cardiac cycle) requires to find the point in a sevendimension space that minimizes an error function.

The purpose of this paper is to propose a new algorithm for the detection of T-wave (detection of maximum or maxima for biphasic waves), as well as for the location of T-wave end, based on a mathematical model given by a skewed Gaussian function, in 12-lead paced ECG signals. The most remarkable difference from existing algorithms (specifically algorithms based on mathematical models) is its innovative and consistent method which makes use of a simple mathematical function, manipulated for skewness, in order to model the T-wave behaviour. Its performance is evaluated using a database of manually annotated 12-lead paced ECG signals recorded during an electrophysiology study of patients with ischemic cardiomyopathy (ICM) undergoing risk stratification for implantable cardioverter defibrillator at the University Hospitals of Leicester National Health Service Trust, Leicester, UK [2]. Additionally the suitability of the method is also evaluated using all the publicly available records of the annotated QT database [27]. 


\section{Materials and Methods}

This paper considers only T-wave peak and T-wave end detections. Obviously, the R-wave and QRS onset and offset need to be accurately detected previously in order to delimit an interval that contains the T-wave. We have adopted our already validated QRS detection and segmentation approach $[1,16]$, which is based on Hilbert and Wavelet transforms, first-derivative and adaptive threshold technique.

Let us first consider monophasic T-waves (positive or negative). Biphasic T-wave can be treated as a particular case of this one, as described in the following section.

\subsection{T-wave modelling: Skewed Gaussian Function}

The different stages of the T-wave detection and $\mathrm{T}$-wave end location algorithm are illustrated in Figure 1. As a starting point, a zero-phase band-pass FIR filter using a Hamming window is applied to each interval between a given QRS offset (J-point) and the subsequent QRS onset (which is named $W[n]$ ) for noise attenuation while preserving the essential spectral content of the T-wave. To avoid the end effects of the digital filter, we consider an extra offset of 50ms in the edges of the input signal. The cut-off frequencies were selected as $[0.01,12] \mathrm{Hz}$ based on the experimental observation that $\mathrm{T}$ wave spectral content was concentrated within the range [2.5, 4] Hz (the upper frequency of the passband allows for up to three harmonics of $4 \mathrm{~Hz}$ ). For more explanations, see the Appendix.

The order of the FIR filter was experimentally selected as 32. Then, inside the training stage, a mathematical modelling is proposed for T-wave obtaining a set of two numerical parameters (standard deviation of a Gaussian function $\sigma$ and distortion angle $\alpha$ ) that characterise the T-wave. After determining the optimal skewed Gaussian function that best models T-wave waveform, crosscorrelation function is applied between the original waveform and the proposed kernel to identify the T-wave peak. Based on some reference points firstly detected in the optimal skewed Gaussian function and, then, mapped into the original waveform, an algorithm based on Trapezium area approach [4] is applied to identify each T-wave end.

Before the explanation of the training stage, we will introduce the proposed method to insert distortion in the common Gaussian function, which will provide a model for the T-wave. Given a Gaussian function $G(x)$ with its maximum amplitude multiplied by a constant $A_{G}$, defined in the interval $x_{G 1} \leq x \leq x_{G 2}$, with standard deviation $\sigma_{1}$, and given a linear function $f(x)$ passing through the origin and with a slope $\alpha$, we derive a resulting function $G^{\prime}(x)$, where

$$
\begin{aligned}
& G(x)=A_{G} \frac{1}{\sqrt{2 \pi} \sigma_{1}} \cdot \exp \left(\frac{-x^{2}}{2 \sigma_{1}^{2}}\right), \\
& G^{\prime}(x)=G(x-\rho(x)), \text { and } \\
& \rho(x)=f(G(x))=\tan (\alpha) \cdot G(x) .
\end{aligned}
$$

As we can see in the example illustrated in Figure 2, where $A_{G}=10,\left[x_{G 1}, x_{G 2}\right]=[-3,3], \sigma_{1}=0.8$ and $\alpha$ $=\pi / 40$, this method produces an asymmetric function $G^{\prime}(x)$ from the Gaussian function $G(x)$. 


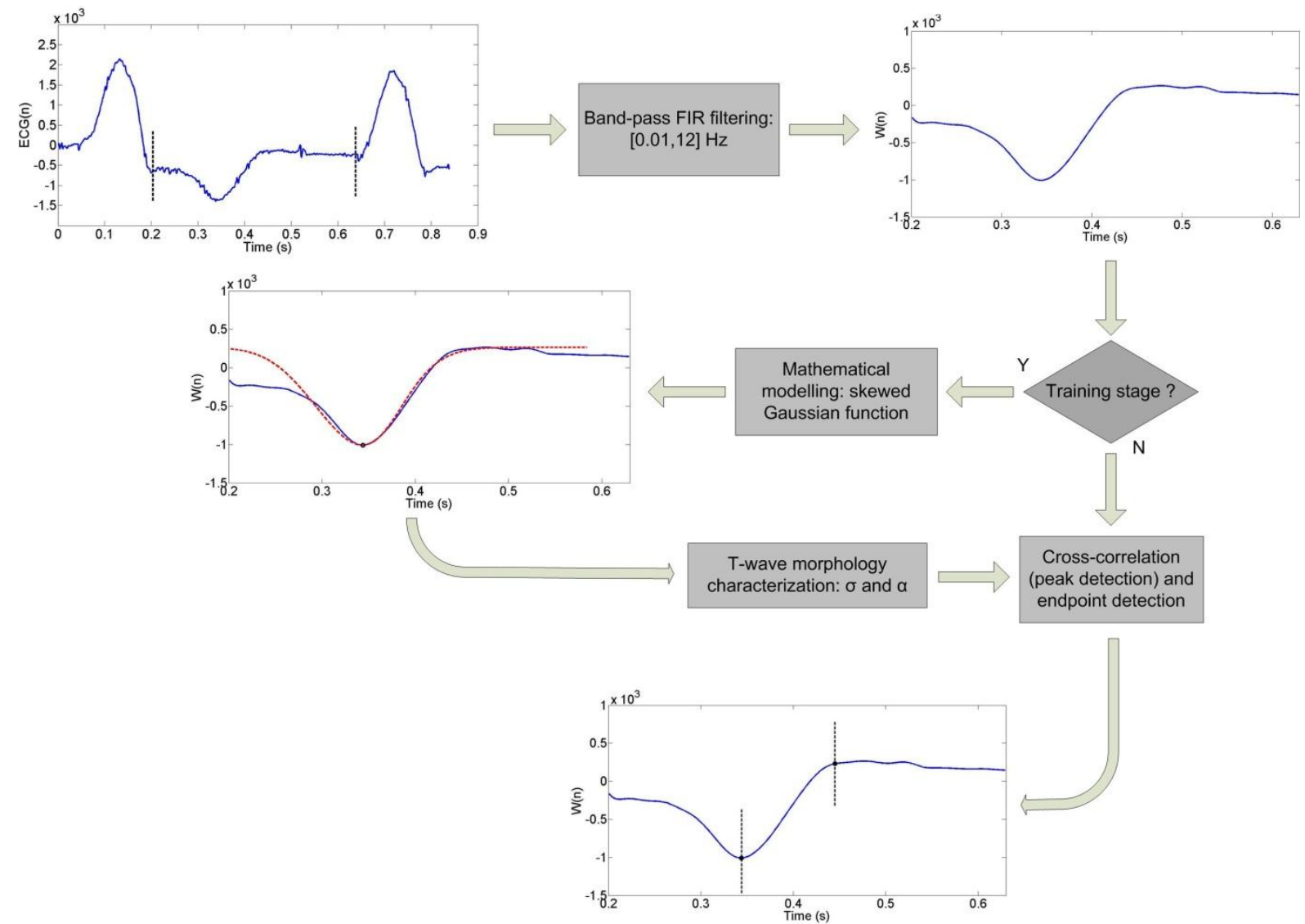

Figure 1. Overall view of the T-wave detection and T-wave end location algorithm.

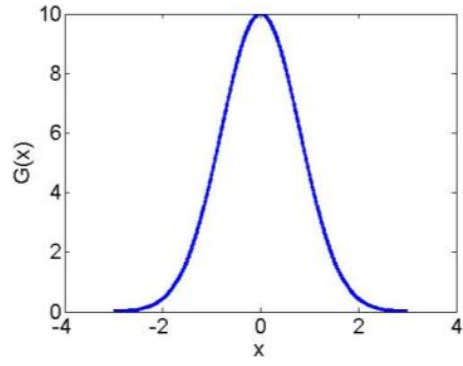

(a)

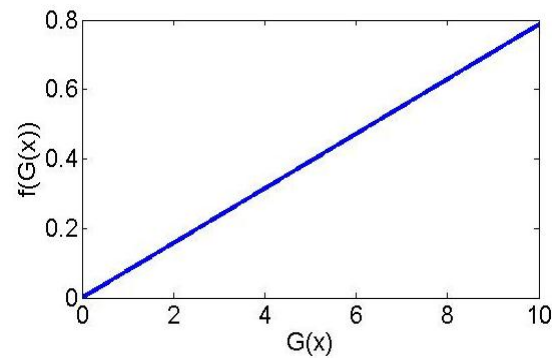

(b)

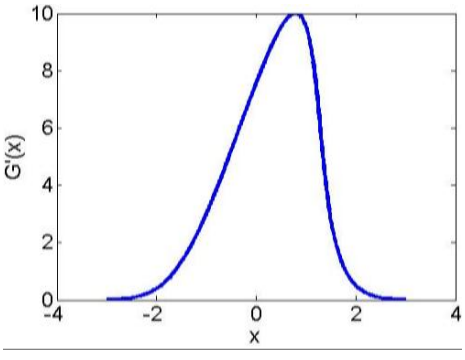

(c)

Figure 2. Proposed process for introducing distortion to a Gaussian function: a) Gaussian function $G(x)$ with standard deviation $\left(\sigma_{1}\right) 0.8$, b) linear function $f(x)=f(G(x))$ with slope $(\alpha) \pi / 40$ and c) resultant skewed Gaussian function $G^{\prime}(x)$.

The Gaussian distortion or skewness is linearly dependent on its amplitude and for each amplitude point it is linearly dependent to the slope angle $\alpha$. For the case shown in Figure 2, we have introduced a skewness that pulls the Gaussian function to the right, but we can also define a negative angle $\alpha$, such that we can pull the Gaussian function to the left.

Concerning the training stage, for each one of the earliest $N$ intervals between each QRS onset and subsequent QRS offset, we apply the cross-correlation function between the corresponding signal window $W[n]$ and a set of skewed Gaussian functions. Each original Gaussian function $G(x)$ is defined in the interval $x_{G 1} \leq x \leq x_{G 2}$, with a resolution equal to $N_{\mathrm{G}}$ (total number of samples) and standard deviation $(\sigma)$ defined in the interval $\sigma_{1} \leq \sigma \leq \sigma_{2}$. The set of distortion angles $(\alpha)$ are defined in 
the interval $\alpha_{1} \leq \alpha \leq \alpha_{2}$. Naming each skewed Gaussian function as $G_{\sigma, \alpha}^{\prime}[n]$, we obtain the resultant filtered signal $S[n]$ as

$$
S[n]=\sum_{\tau=0}^{K} W[\tau] \cdot G_{\sigma, \alpha}^{\prime}[n-\tau]
$$

The location of the maximum amplitude of $|S[n]|, P_{\alpha \sigma}^{(1)}$, is determined. For each signal window $W[n]$, an error function $E_{\sigma, \alpha}^{1}$ is computed through the following sequence of steps:

- We take a subset of the samples of $W[n]$, given by $W_{\mathrm{s}}[k], P_{\alpha \sigma}^{(1)}-\gamma_{1} \leq k \leq P_{\alpha \sigma}^{(1)}+\gamma_{2}$, where $\gamma_{1}$ and $\gamma_{2}$ are offset times.

- Then, the skewed Gaussian function $G_{\sigma, \alpha}^{\prime}[n]$ is normalised according to the maximum and minimum values of $\left|W_{\mathrm{s}}[k]\right|$ and aligned by its peak amplitude location with $P_{\alpha \sigma}^{(1)}$.

- Naming the resulting shifted and normalised skewed Gaussian function as $G_{\sigma, \alpha}^{\prime \prime}[k]$, $P_{\alpha \sigma}^{(1)}-\gamma_{1} \leq k \leq P_{\alpha \sigma}^{(1)}+\gamma_{2}$, we obtain $E_{\sigma, \alpha}^{(1)}$ as

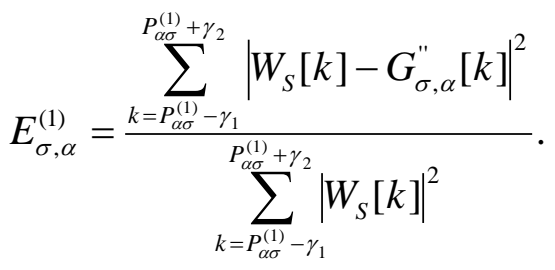

After computing $E_{\sigma, \alpha}^{(1)}$ for all the possible combinations of the pair $(\sigma, \alpha), \sigma_{1} \leq \sigma \leq \sigma_{2}$ and $\alpha_{1} \leq \alpha \leq \alpha_{2}$, we find the particular pair $\left(\sigma_{\mathrm{s}}, \alpha_{\mathrm{s}}\right)$ which corresponds to the lowest value of $E_{\sigma, \alpha}^{(1)}$, for each signal window $W[n]$. Then, naming the location $P_{\alpha \sigma}^{(1)}$ found with $\left(\sigma_{\mathrm{s}}, \alpha_{\mathrm{s}}\right)$ as $P^{(2)}$, we obtain another set of values for an error function $E_{\sigma, \alpha}^{(2)}$. As we had done for the computing of $E_{\sigma, \alpha}^{(1)}$, we normalise and align the skewed Gaussian functions according to the maximum and minimum values of $\left|W_{\mathrm{s}}[k]\right|$, $P^{(2)}-\gamma_{1} \leq k \leq P^{(2)}+\gamma_{2}$, and obtain

$$
E_{\sigma, \alpha}^{(2)}=\frac{\sum_{k=P^{(2)}-\gamma_{1}}^{P^{(2)}+\gamma_{2}}\left|W_{S}[k]-G_{\sigma, \alpha}^{\prime \prime}[k]\right|^{2}}{\sum_{k=P^{(2)}-\gamma_{1}}^{P^{(2)}+\gamma_{2}}\left|W_{S}[k]\right|^{2}} .
$$

The optimal parameters $\left(\sigma_{0}, \alpha_{0}\right)$ which model a given T-wave are the ones that correspond to the lowest value of $E_{\sigma, \alpha}^{(2)}$. We average the individual values related to each one of the T-waves pertaining to the training set to obtain $\overline{\sigma_{o}}$ and $\overline{\alpha_{o}}$. The average of the minimum values obtained for $E_{\sigma, \alpha}^{(2)}$, related to each T-wave, is stored and named as $E_{\min }^{(2)}$. 
The optimal parameters $\overline{\sigma_{o}}$ and $\overline{\alpha_{o}}$ are used to characterise the skewed Gaussian function $G_{\sigma_{o},}^{\prime}, \overline{\alpha_{o}}[n]$ and model each one of the T-waves throughout the whole ECG signal. The detection of the peak of each T-wave, whose location we name $T_{p}[m], 1 \leq m \leq M, M$ being the total number of Twaves, is performed by computing the cross correlation function

$S[n]=\sum_{\tau=0}^{K} W[\tau] \cdot G_{\overline{\sigma_{o}}, \overline{\alpha_{o}}}^{\prime}[n-\tau]$

and locating the maximum amplitude of $|S[n]|$. If, at any time, the associated error function (see equation 5) between the corresponding signal window and $G^{\prime \prime}{\overline{\sigma_{o}}}_{\overline{\alpha_{o}}}[k]$ (shifted and normalised skewed Gaussian function), $T_{p}[m]-\gamma_{1} \leq k \leq T_{p}[m]+\gamma_{2}$, is higher than $\theta \cdot E_{\min }^{(2)}$, where $\theta$ is a tolerance factor, then we proceed with another learning process for this specific signal window. For the other signal intervals, we keep the parameters $\overline{\sigma_{o}}$ and $\overline{\alpha_{o}}$.

We illustrate in Figure 3 different T-wave morphologies (continuous line) with the proposed skewed Gaussian functions (dashed line) after determining the corresponding optimal parameters $\overline{\sigma_{o}}$ and $\overline{\alpha_{o}}$. The detected T-wave peaks are already highlighted.

\subsection{The issue of biphasic T-waves}

Concerning biphasic T-waves, the proposed skewed Gaussian function, which is essentially a monophasic function, is able to model the T-wave phase with the highest energy in the training stage. However, for biphasic T-waves the predominance of each phase can change, throughout the ECG signal (especially in paced ECGs). Therefore, the following simple rule is adopted. In the analysis stage, we search for the local maxima or minima in each filtered signal $S[n]$ (equation 7) whose absolute values exceed $70 \%$ of the maximum value of $|S[n]|$ (experimentally selected threshold). Then, if we find a local maximum and a local minimum, we test if the optimal skewed Gaussian function is positive or negative, and we choose as the T-wave peak the sample with the same amplitude sign as the optimal skewed Gaussian function.

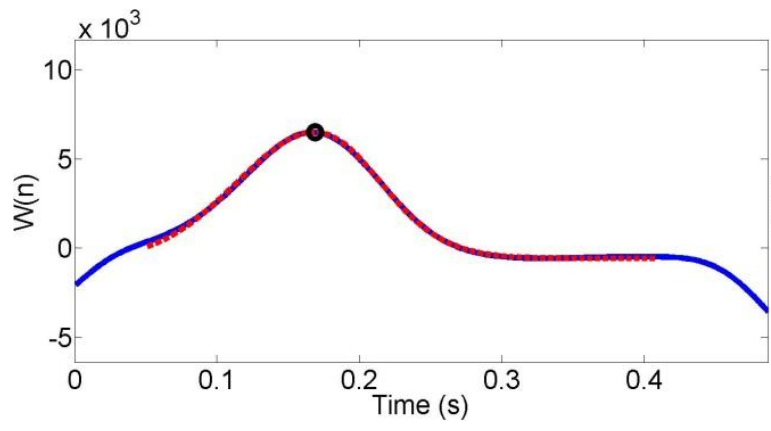

(a)

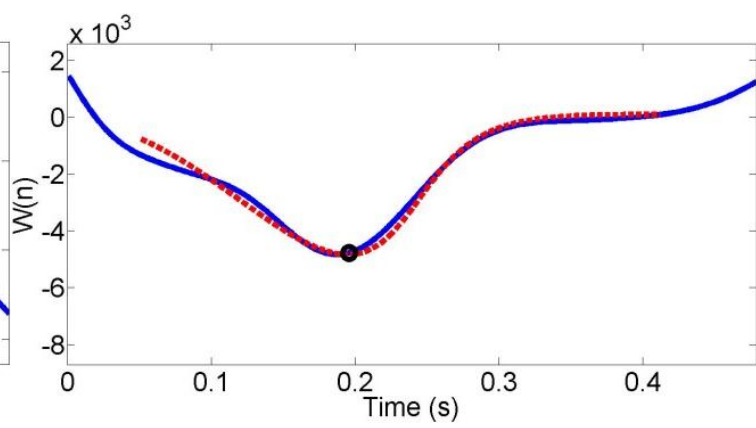

(b) 


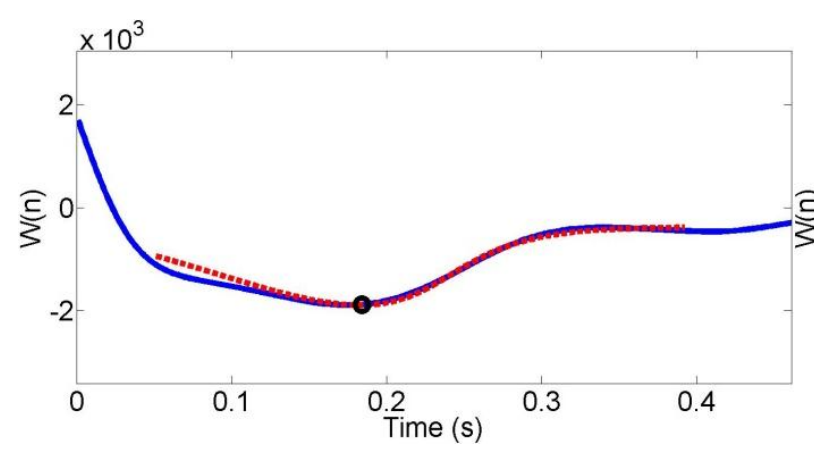

(c)

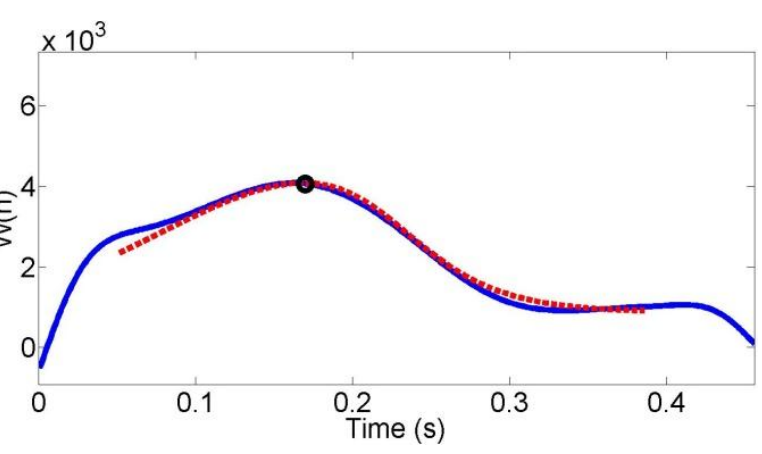

(d)

Figure 3. Examples of different T-wave morphologies (continuous line) with the optimal skewed Gaussian functions (dashed line) modelling each waveform and the corresponding T-wave peaks detected.

\subsection{T-wave end detection: combination of approaches}

Before proceeding with the algorithm for $\mathrm{T}$-wave end detection, we need to figure out the wave morphology, e.g. determine in an automatic way if it is monophasic or biphasic. For each signal window $W[n]$, we compute for each instant $k$ inside the referred window [11]

$$
\begin{aligned}
& \overline{s_{k}}=\frac{1}{2 p+1} \sum_{j=k-p}^{k+p} W[j] \text { and } \\
& A_{k}=\sum_{j=k-w+1}^{k}\left(W[j]-\overline{s_{k}}\right),
\end{aligned}
$$

where $w$ (sliding window) is equal to the number of samples corresponding to the time duration of $128 \mathrm{~ms}$ and $p$ (smoothing window) is equal to the number of samples corresponding to the time duration of $16 \mathrm{~ms}$ [11].

Then, we find $k_{1}$ and $k_{2}$, respectively, as the locations where we have the maximum and minimum values of $A_{k}$ inside $W[n]$. For a given parameter $\lambda$, we test the condition [11]

$$
\frac{1}{\lambda}<\frac{\left|A_{k_{1}}\right|}{\left|A_{k_{2}}\right|}<\lambda,
$$

such that, if it is true, then we have biphasic T-wave. Otherwise, we have monophasic T-wave.

In the case of monophasic T-wave, the following sequence of steps is performed:

- We search in the optimal skewed Gaussian function for the location of the sample with the maximum absolute value of the first-derivative function after the peak location. This position and the corresponding absolute value of the first derivative function are named $T_{d e r}$ and $M_{d e r}$.

- Afterwards, we search also in the referred kernel for the location of the first sample after $T_{d e r}$ whose magnitude of the first-derivative is below $\beta_{1} \cdot M_{d e r}$, where $\beta_{1}$ is a percentage factor.The corresponding position is named $T_{\min }$. 
- These two obtained locations are mapped into the signal window $W[n]$ and we apply the Trapezium area approach, proposed by Vázquez-Seisdedos et al. [4].

Basically, we define a moving sample, $T_{e}$, where $T_{d e r} \leq T_{e} \leq T_{\min }$. The vertices of the trapezium are defined by the following pairs $(x, y):\left(T_{d e r}, W\left[T_{d e r}\right]\right),\left(T_{e}, W\left[T_{e}\right]\right),\left(T_{\min }, W\left[T_{e}\right]\right)$ and $\left(T_{\min }, W\left[T_{d e r}\right]\right)$. The area of the trapezium is given as:

$$
A_{T}\left(T_{e}\right)=0.5\left(W\left[T_{d e r}\right]-W\left[T_{e}\right]\right)\left(2 T_{\min }-T_{e}-T_{d e r}\right) .
$$

Then, T-wave end is defined as the point where the area $A_{T}\left(T_{e}\right)$ is maximum, and we name its location $T_{\text {end }}[m], 1 \leq m \leq M, M$ being the total number of T-waves.

As an illustrative example, for a given search window $W[n]$, the detection of the referred samples locations $T_{d e r}$ and $T_{\min }$ is illustrated in Figure 4(a). The computing of the trapezium's area $A_{T}\left(T_{e}\right)$, its corresponding behaviour with the maximum point $A_{T}\left(T_{\text {end }}\right)$ and, finally, the location of the T-wave end are shown, respectively, in Figures 4(b), 4(c) and 4(d).

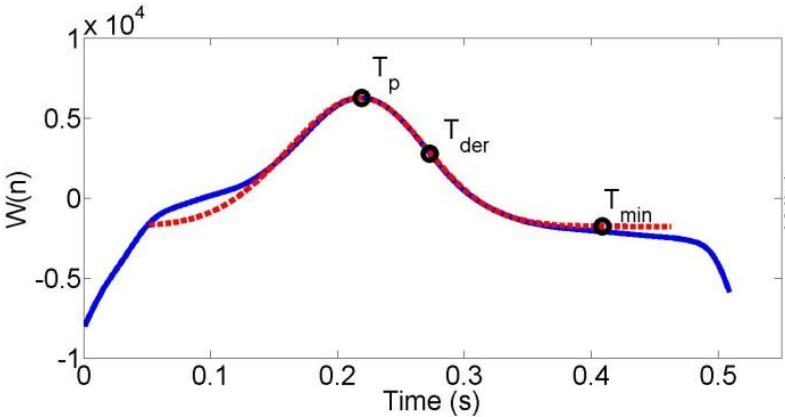

(a)

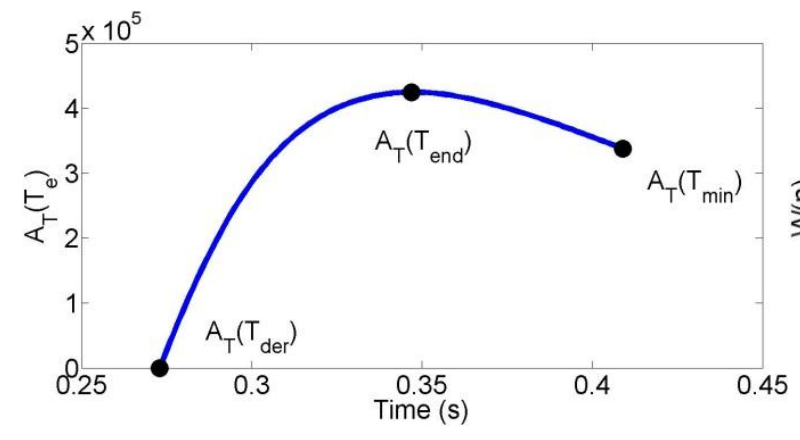

(c)

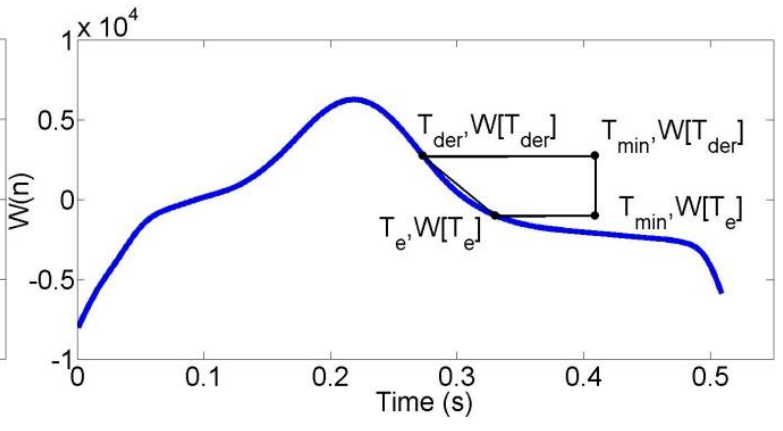

(b)

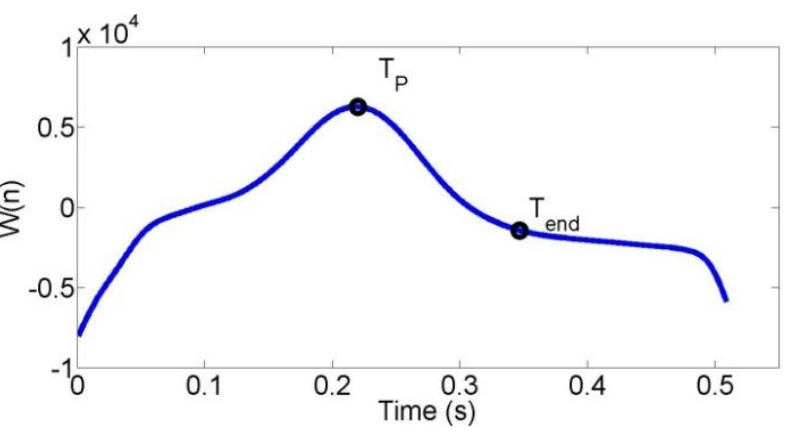

(d)

Figure 4. Process to identify T-wave end: (a) detection of the samples locations $T_{d e r}$ and $T_{\min }$ in the skewed Gaussian function (dashed line) and their projection upon the T-wave search window (continuous line), (b) designing of the trapezium's area approach, (c) computing of $A_{T}\left(T_{\mathrm{e}}\right)$ with its maximum occurring in the T-wave end and (d) location of the sample related to T-wave end.

In the case of biphasic T-waves, we firstly verify which phase is modelled by the optimal skewed Gaussian function. For this we test if the obtained kernel is positive or negative and, then, we determine if $k_{1}$ is located before $k_{2}$ or vice-versa (see equations 8,9 and 10). If the optimal skewed Gaussian function models the second phase of the biphasic T-wave, then the process to identify the T- 
wave end follows the same sequence of steps explained for monophasic T-waves. Otherwise, we proceed with the following sequence of steps:

- We select other window which confines the peak and the end of the second phase, $W_{2}[n]$. Considering the optimal skewed Gaussian function which models the first phase of the T-wave, the beginning of $W_{2}[n]$ is defined as the position $T_{d e r}$.

- The end of $W_{2}[n]$ is defined as the subsequent QRS offset.

- Then, we search inside $W_{2}[n]$ for the sample related to the peak (local maximum or local minimum) of the second phase, $T_{p}^{(2)}$, which may be located before the latest location among $k_{1}$ and $k_{2}$.

- After that we search for the sample located after $T_{p}^{(2)}$ which is related to the local maximum (magnitude) of the first-derivative function. This position and the corresponding absolute value of the first derivative function are named $T_{d e r}^{(2)}$ and $M_{d e r}^{(2)}$.

- Analogously as implemented for monophasic T-waves, we detect the first sample whose absolute value of the first-derivative function is below $\beta_{2} . M_{d e r}^{(2)}$, where $\beta_{2}$ is a percentage factor. We name the corresponding position $T_{\min }^{(2)}$. For its detection, we consider a time threshold given as $100 \mathrm{~ms}$ after $T_{p}^{(2)}$.

- Finally, we compute the Trapezium area expression $A_{T}\left(T_{e}^{(2)}\right)$, now considering $T_{e}^{(2)}$ as the moving sample, where $T_{d e r}^{(2)} \leq T_{e}^{(2)} \leq T_{\min }^{(2)}$. T-wave end is defined as the point where the area $A_{T}\left(T_{e}^{(2)}\right)$ has maximum value.

As an example of the application of the proposed approach, a T-wave search window containing a biphasic waveform and the skewed Gaussian function which models its first phase are shown in Figure 5(a). The window $W_{2}[n]$ and the computing of the trapezium area expression for the second phase are presented in Figure 5(b). As illustrated in Figures 5(c) and 5(d), T-wave end is located as the sample related to the maximum value of $A_{T}\left(T_{e}^{(2)}\right)$.

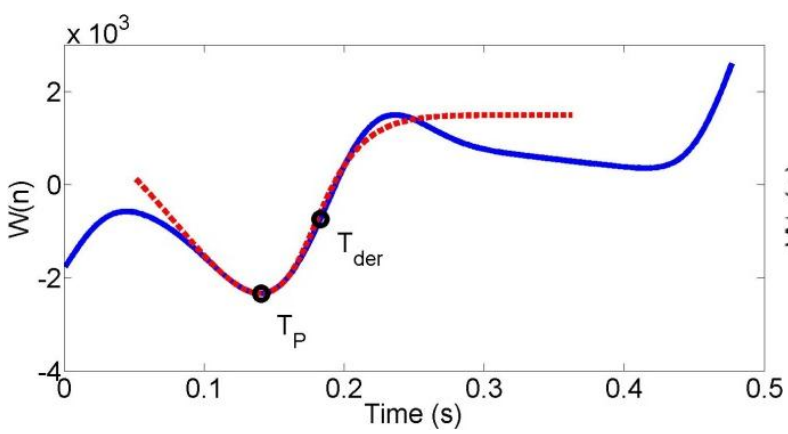

(a)

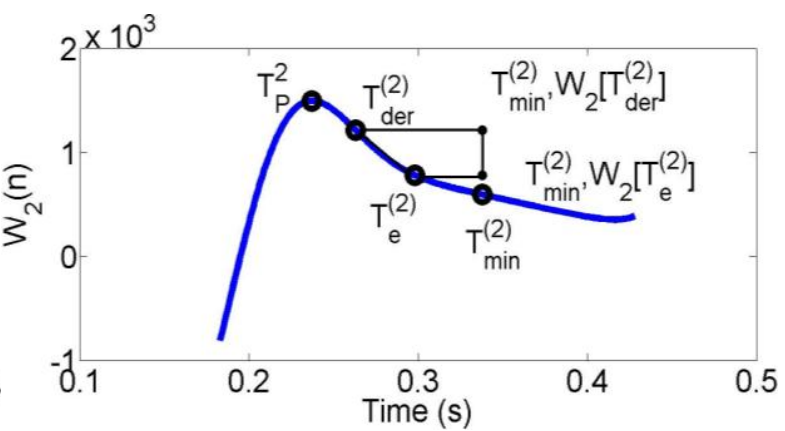

(b) 


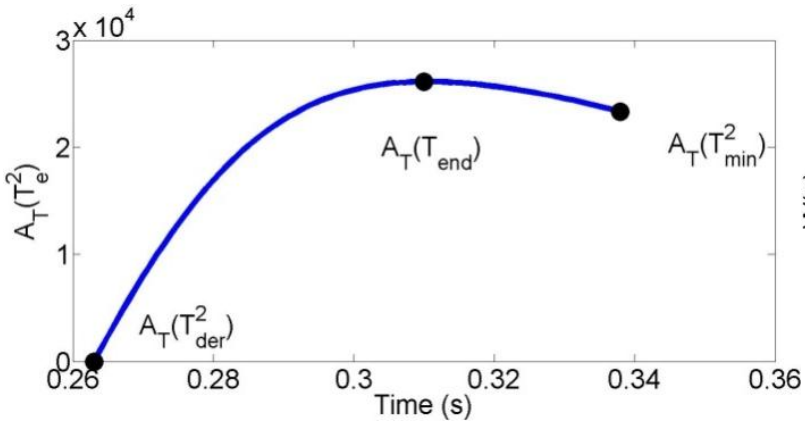

(c)

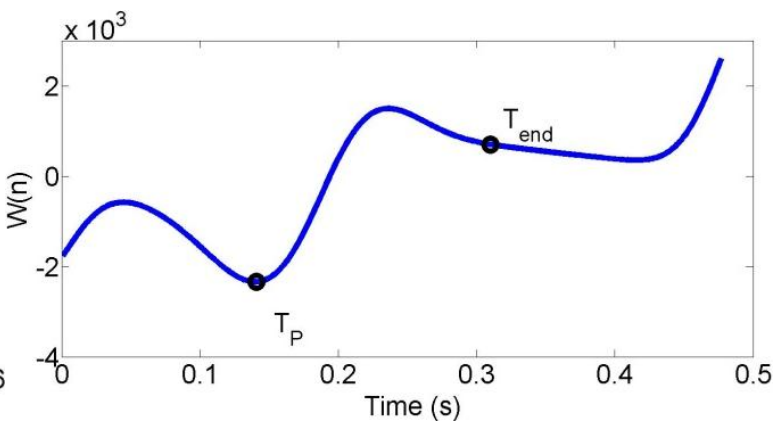

(d)

Figure 5. Process to identify T-wave end in biphasic T-waves: (a) detection of the peak $\left(T_{P}\right)$ and the subsequent location of the maximum first-derivative $\left(T_{d e r}\right)$ of the first phase, (b) designing of the Trapezium area approach with detection of the peak $T_{P}{ }^{(2)}$, the subsequent maximum derivative $\left(T_{d e r}{ }^{(2)}\right)$ and subsequent minimum derivative $\left(T_{\min }{ }^{(2)}\right)$ of the second phase, (c) computing of $A_{T}\left(T_{e}^{(2)}\right)$ with its maximum occurring in the T-wave end and (d) location of the sample related to T-wave end.

\subsection{Databases and Validation Methods}

\section{A. Database of 12-lead paced ECG signals}

For the validation of the proposed approach, we used a set of 12-lead paced ECG signals related to 13 patients, which were collected in the University Hospitals of Leicester National Health Service Trust, UK, containing a total of 4680 annotated T-wave peaks and ends. Regarding the set of patients, all of them had undergone an electrophysiology study (EPS) between $1^{\text {st }}$ January 2010 and $31^{\text {st }}$ March 2012. Ten patients present a history of ischemic cardiomyopathy, and for them the EPS was part of clinical risk stratification for ICD implantation. The other three had an electrophysiology study for diagnosis and treatment of supraventricular tachycardia. This database was part of another research project which aimed to prospectively investigate a new electrophysiological measure of electrical restitution heterogeneity for predicting risk of ventricular arrhythmia/death in patients with ischemic cardiomyopathy who were candidates for ICD therapy [2]. Concerning that study, ethical approval was granted by the Derbyshire Research Ethics Committee and the study protocol was approved by the Research and Development Office of the University Hospitals of Leicester National Health Service Trust, UK. All patients gave written, informed consent.

The standard 12-lead electrocardiograms were recorded at $1 \mathrm{kHz}$ sampling rate, with a high pass filter set to $0.01 \mathrm{~Hz}$ and a low pass filter set to $50 \mathrm{~Hz}$, and exported at 16-bit digital resolution. The medical study protocol used a 10-beat train at drive cycle lengths of $600 \mathrm{~ms}$ and $400 \mathrm{~ms}$. A single extrastimulus protocol was followed with decrements of $20 \mathrm{~ms}$ to $300 \mathrm{~ms}$ and then $10 \mathrm{~ms}$ to effective refractory period. For the last two beats of each drive train and the extra stimulus (considering all the 12 leads of each patient), the timing of QRS onset, T-wave peak and T-wave end were manually identified by a senior electrophysiology research fellow from the Department of Cardiovascular Sciences of the University of Leicester. For each analysed T-wave, we process each lead independently and compute the time difference (error) between the automatic detections and the manual annotations for both T-wave peak and T-wave end. The detection of each T-wave was also evaluated for each signal lead by computing the sensitivity, $S e$, the positive predictivity, $P^{+}$, and the failed detection percentage, $F_{d}$. 


\section{B. Database from PhysioNet: QT database}

Additionally, we evaluated the algorithms using the publicly available QT database (QTDB). The QTDB was developed for wave limit validation purposes with a total of 105 records and with two leads, each of them with a length of 15 minutes and $250 \mathrm{~Hz}$ sampling frequency. It provides annotations, by an expert cardiologist, for at least 30 beats per recording, with marks including QRS complexes, P and T wave peaks, onsets and offsets. QTDB includes some recordings from European ST-T database, MIT-BIH Arrhythmia database and several other MIT-BIH databases (ST Change, Supraventricular Arrhythmia, Normal Sinus Rhythm, Sudden Death and Long Term) [27].

\section{Results and Validation}

The suggested values for the whole set of parameters needed for the proposed system, which were determined based on extensive experimental tests, are presented in Table 1, divided in two groups: training stage parameters and analysis stage parameters. Only the Gaussian resolution $\left(N_{\mathrm{G}}\right)$ is adapted according to the sampling frequency of the analysed ECG signals.

Table 1. Set of parameters and their respective values.

\begin{tabular}{|l|l|}
\hline Training stage parameters & Values \\
\hline $\mathrm{N}$ (beats for training stage) & 5 \\
\hline$\left[\sigma_{1}, \sigma_{2}\right]$ & {$[0.3,1.2]$} \\
\hline$\left[\alpha_{1}, \alpha_{2}\right]$ & {$[0, \pi / 20]$} \\
\hline $\mathrm{A}_{\mathrm{G}}$ & 10 \\
\hline$\left[x_{G 1}, x_{G 2}\right]$ & {$[-3,3]$} \\
\hline $\begin{array}{l}\mathrm{N}_{\mathrm{G}} \text { (Gaussian resolution), } 1 \mathrm{kHz} \\
\text { sampling frequency }\end{array}$ & 500 \\
\hline $\begin{array}{l}\mathrm{N}_{\mathrm{G}} \text { (Gaussian resolution), } 250 \\
\mathrm{~Hz} \text { sampling frequency }\end{array}$ & 125 \\
\hline$\gamma_{1}$ & $60 \mathrm{~ms}$ \\
\hline$\gamma_{2}$ & $100 \mathrm{~ms}$ \\
\hline \multicolumn{2}{|l|}{} \\
\hline Analysis stage parameters & Values \\
\hline$\Theta$ & 3 \\
\hline$\lambda$ & 10 \\
\hline$\beta_{1}$ & $1 \%$ \\
\hline$\beta_{2}$ & $5 \%$ \\
\hline
\end{tabular}

Some special cases of $\mathrm{T}$-wave morphologies with the corresponding results for the parameterized skewed Gaussian function as well as for $\mathrm{T}$-wave peak detection and $\mathrm{T}$-wave end location are illustrated in Figures 6(a) - 6(m). Results reported by Figures 6(a) $-6(\mathrm{~h})$ are related to the database of 12-lead paced ECG signals (lead I for all the cases). Whereas Figures 6(i) - 6(q) are related to ECG signals from QT database (various leads). 


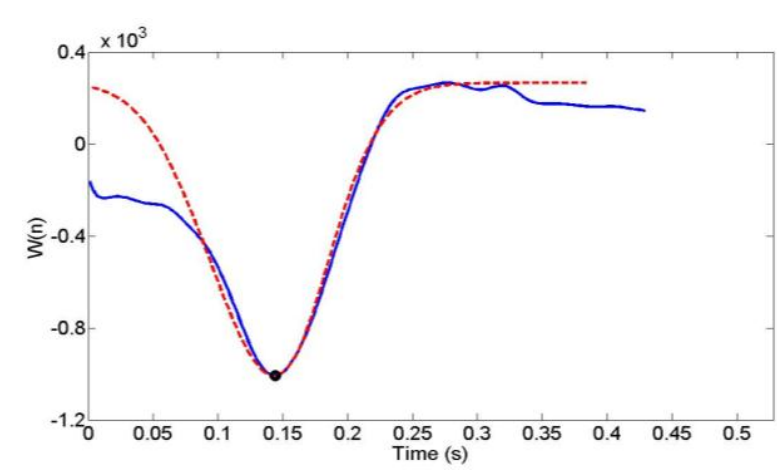

(a)

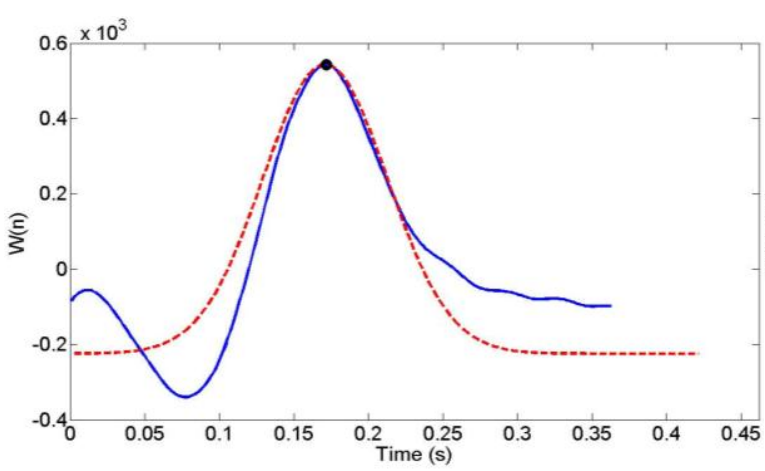

(c)

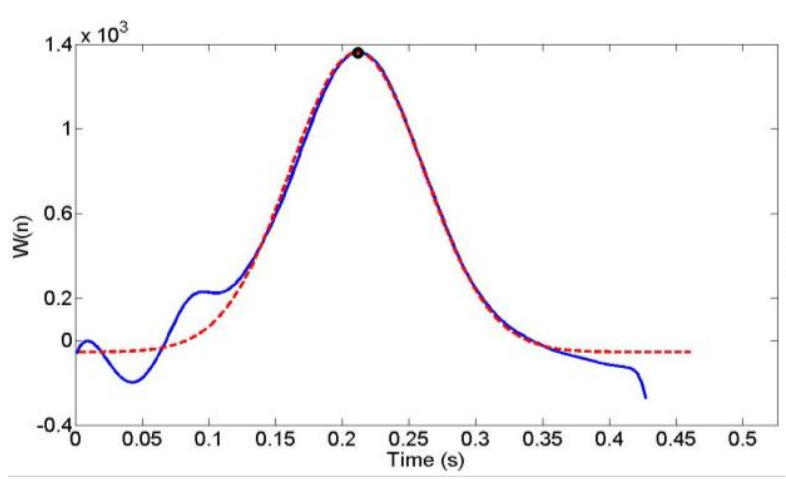

(e)

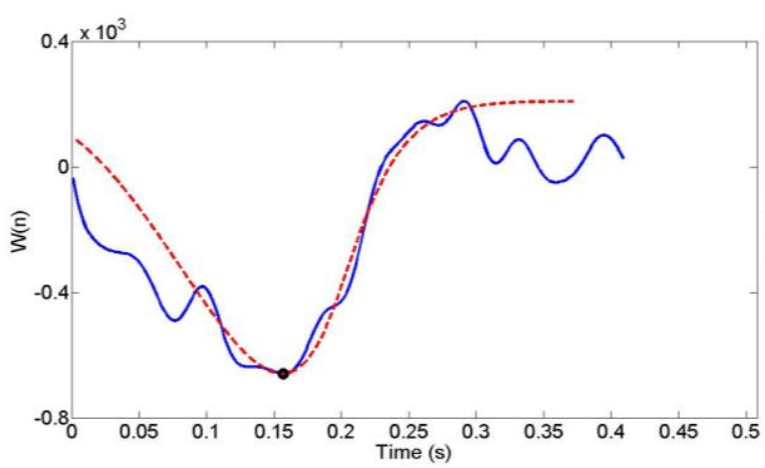

(g)

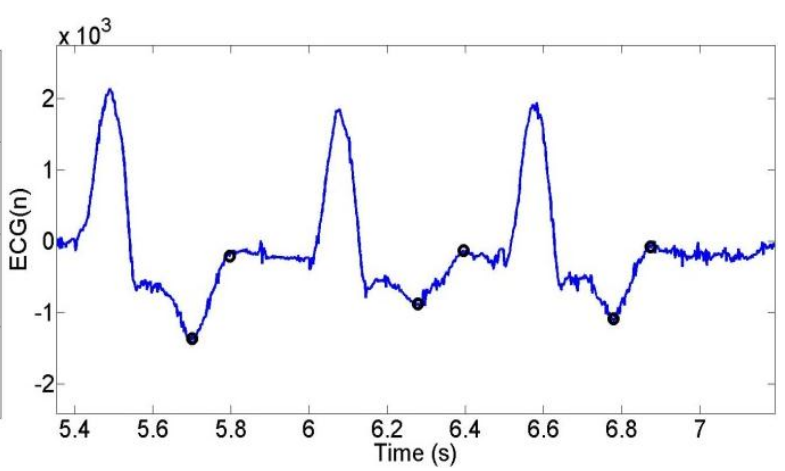

(b)

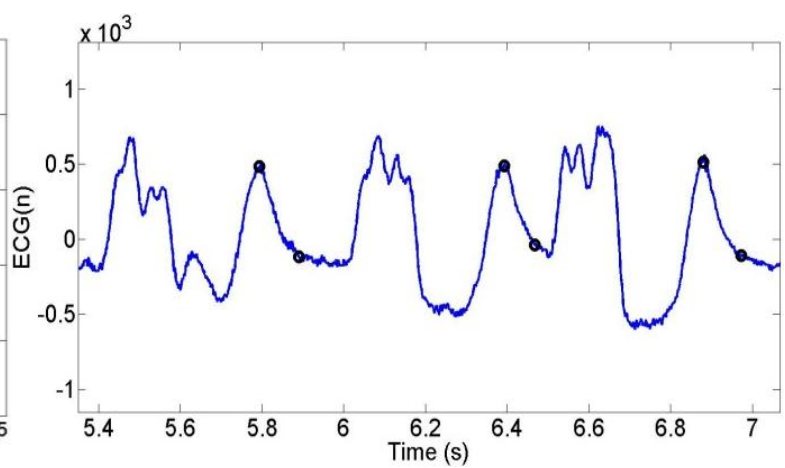

(d)

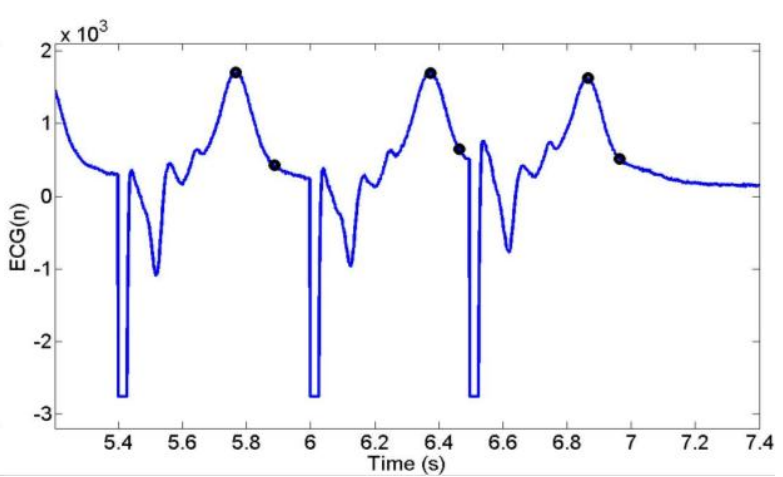

(f)

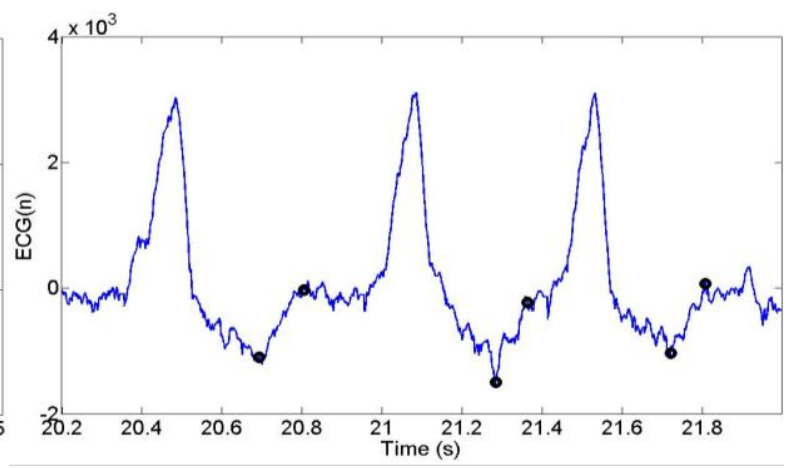

(h) 


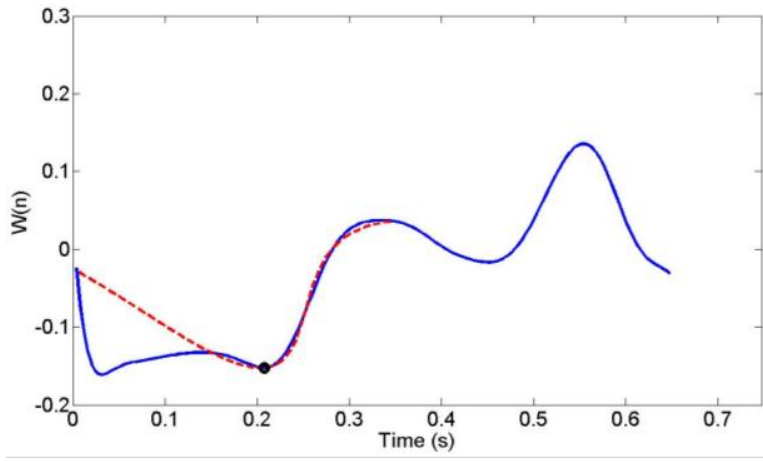

(i)

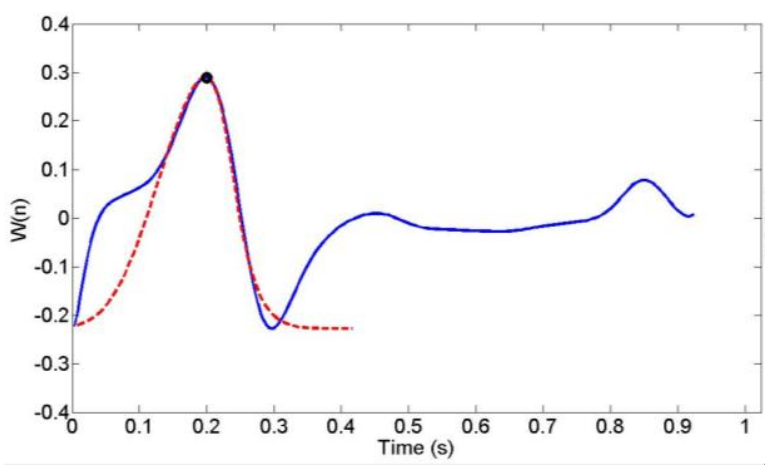

(1)

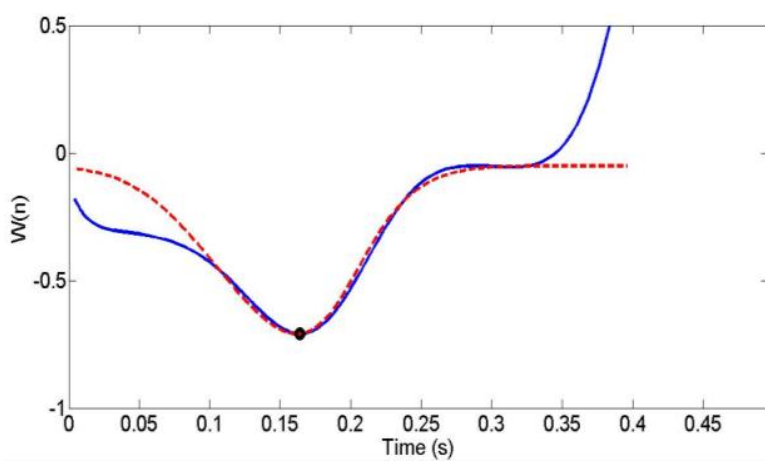

(n)

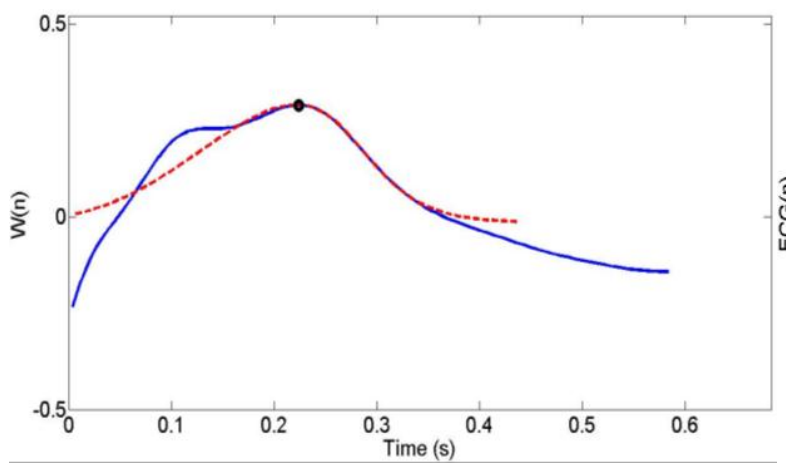

(p)

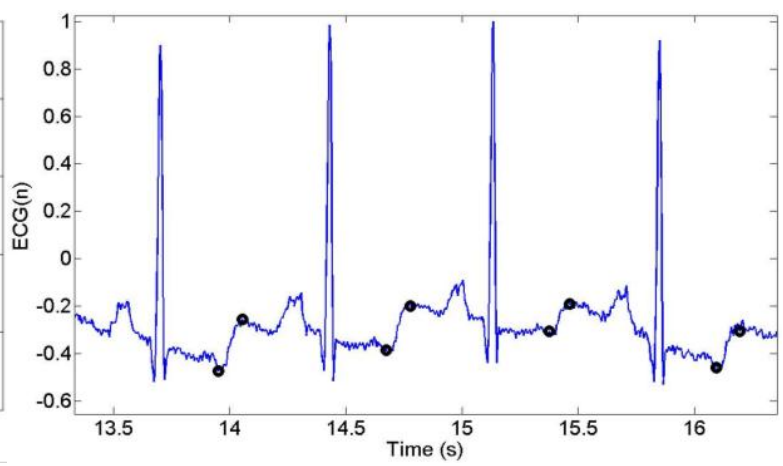

(j)

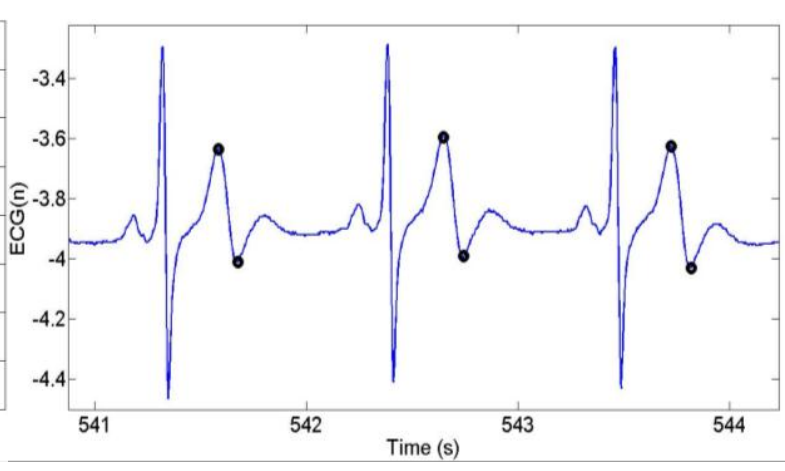

(m)

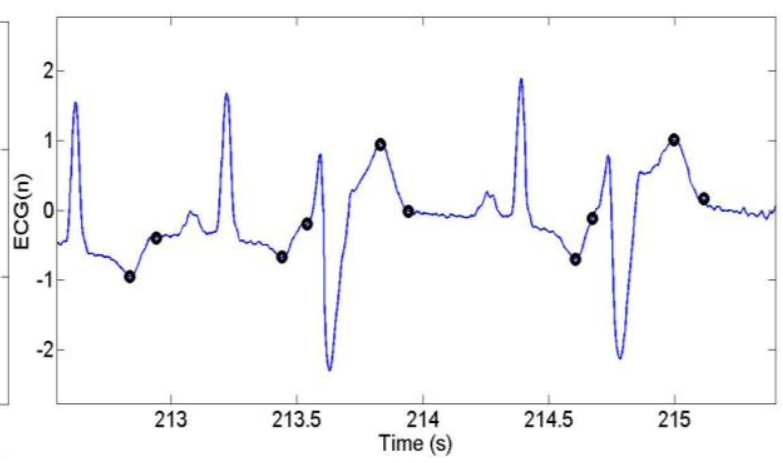

(o)

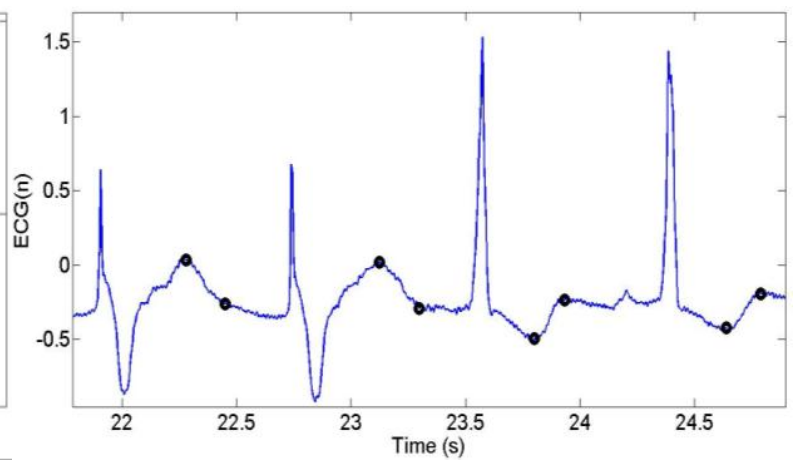

(q)

Figure 6. Results for skewed Gaussian function parameterization as well as for T-wave peak and T-wave end locations for different morphologies: (a) and (b), negative paced T-wave, (c) and (d), biphasic paced T-wave with the peak in the second 
phase, (e) and (f), positive paced T-wave, (g) and (h), negative paced T-wave with low SNR, (i) and (j), ascending nonpaced T-wave, (l) and (m), positive non-paced T-wave, (n) and (o) predominant negative non-paced T-wave morphology and occurrences of premature ventricular contraction, (p) and (q) predominant positive paced T-wave morphology alternating with negative non-paced T-wave morphologies.

\section{A. Results over the database of 12-lead paced ECG signals}

The overall results of the validation for T-wave detection in terms of positive predictivity, sensitivity and failed detection percentage considering all the 13 patients, are illustrated in Table 2 . The overall results for T-wave peak and T-wave end locations in terms of the average and standard deviation $(m \pm$ $s d$ ) of the time errors are illustrated in Table 3. Finally, the detailed results of the average and standard deviation of the time errors for both T-wave peak and T-wave end locations for each lead, considering all the 13 patients, are synthesized in Table 4. The tolerance for the standard deviation of detection time errors for T-wave end given by the Common Standards of Electrocardiography (CSE) Working Party $[15,20,21]$ is presented in the last row of Table 4.

Table 2. Overall results for T-wave detection performance for all patients.

\begin{tabular}{|c|c|c|c|}
\hline Fiducial Point & $\operatorname{Se}(\boldsymbol{\%})$ & $\mathbf{P}^{+}(\boldsymbol{\%})$ & $\mathbf{F}_{\mathbf{d}}(\boldsymbol{\%})$ \\
\hline T-wave peak & 99.12 & 99.12 & 1.76 \\
\hline
\end{tabular}

Table 3. Overall results for T-wave peak and T-wave end locations for all patients (TE: time error).

\begin{tabular}{|c|c|}
\hline Fiducial Point & TE $(\boldsymbol{m} \pm \boldsymbol{s} \boldsymbol{d})(\boldsymbol{m} \boldsymbol{s})$ \\
\hline T-wave peak & $-0.38 \pm 7.12$ \\
\hline T-wave end & $-3.70 \pm 15.46$ \\
\hline
\end{tabular}

Table 4. Performance comparison of $\mathrm{T}$-wave peak and $\mathrm{T}$-wave end locations considering each ECG lead for all patients (TE: time error).

\begin{tabular}{|c|c|c|}
\hline Lead & $\begin{array}{c}\text { T-wave peak: } \\
\text { TE }(\boldsymbol{m} \pm \boldsymbol{s} \boldsymbol{d})(\boldsymbol{m} \boldsymbol{s})\end{array}$ & $\begin{array}{c}\text { T-wave end: } \\
\text { TE }(\boldsymbol{m} \pm \boldsymbol{s} \boldsymbol{d})(\boldsymbol{m} \boldsymbol{s})\end{array}$ \\
\hline I & $-4.17 \pm 18.65$ & $2.44 \pm 33.30$ \\
\hline II & $0.18 \pm 4.95$ & $-3.53 \pm 15.58$ \\
\hline III & $0.73 \pm 5.24$ & $-2.56 \pm 12.44$ \\
\hline aVR & $-1.15 \pm 8.91$ & $1.16 \pm 18.89$ \\
\hline aVL & $-2.37 \pm 15.10$ & $-3.59 \pm 19.20$ \\
\hline aVF & $2.32 \pm 8.03$ & $-5.69 \pm 16.86$ \\
\hline V1 & $-0.56 \pm 4.13$ & $-2.59 \pm 10.33$ \\
\hline V2 & $0.12 \pm 2.97$ & $-2.57 \pm 12.00$ \\
\hline V3 & $0.10 \pm 1.12$ & $-4.13 \pm 10.06$ \\
\hline V4 & $0.04 \pm 2.47$ & $-4.66 \pm 9.76$ \\
\hline V5 & $0.40 \pm 2.85$ & $-6.29 \pm 9.45$ \\
\hline V6 & $-0.83 \pm 10.64$ & $-4.38 \pm 14.68$ \\
\hline Tolerances $\left(\mathbf{2} \mathbf{s}_{\mathbf{C S E}}\right)(\mathbf{m s})$ & $\mathbf{3 0 . 6}$ \\
\hline
\end{tabular}


In order to provide comparison of the detailed evaluation results for each one of the twelve ECG leads, we have also analysed the differences considering $\mathrm{T}$-wave power throughout the different leads. For this, we have computed the signal-to-noise ratio (SNR) inside the T-wave search windows of each lead for every patient, considering that the same band-pass filter used at this approach (see section IIA) provides signal denoising. Firstly, we average the results of each lead for each individual patient. Then we compute the median for the set of average results related to each lead over all patients, as well as the $25^{\text {th }}$ and $75^{\text {th }}$ percentiles. We illustrate the results of this analysis in Figure 7 using a box plot in which the central mark is the median, the edges of each box are the $25^{\text {th }}$ and $75^{\text {th }}$ percentiles and the whiskers extend to the most extreme observed values, excepting for outliers, which are represented by markers ' + '. As we can see, lead I and lead aVL presented the lowest median values at $23.8 \mathrm{~dB}$ and $22.2 \mathrm{~dB}$, respectively, and the highest data dispersion. Lead I presented the lowest 'minimum' and the lowest 'maximum' observed values, respectively, at $1.7 \mathrm{~dB}$ and $26.4 \mathrm{~dB}$. Leads V2-V5 presented the highest median values, all above $27 \mathrm{~dB}$, and the lowest data dispersion. Lead V4 presented the highest 'minimum' and the highest 'maximum' observed values, respectively, at 22.4 $\mathrm{dB}$ and $28 \mathrm{~dB}$.

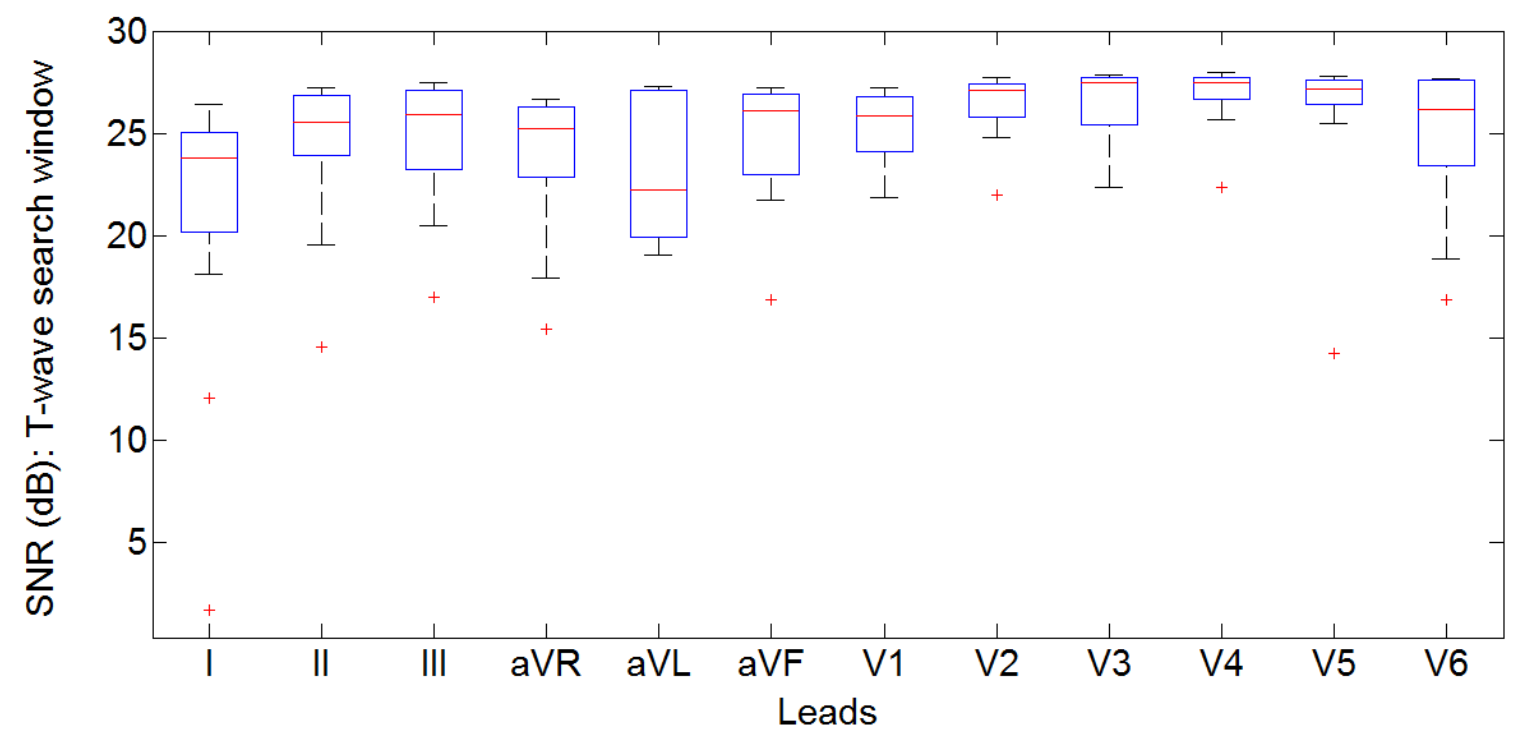

Figure 7. Analysis using box plot of Signal-to-Noise ratio (SNR) inside T-wave search segments for each lead considering all patient data using 12-lead paced ECG signals.

\section{B. Results over QT database}

The global validation results of T-wave detection rates, sensitivity and positive predictivity, over all the publicly available records, using both the available channels, as well as the overall average and standard deviation of T-wave peak and T-wave end time locations errors obtained by our approach and by other five different segmentation algorithms are shown in Table 5. Considering that the manual annotation process of T-wave peaks and ends for QTDB was performed having in sight each pair of available leads, we choose for each point the channel with less error, as recommended by Martínez et al. [15] and Zhang et al. [11]. 
Table 5. Performance comparison of T-wave detection rates, $\mathrm{T}$-wave peak and $\mathrm{T}$-wave end time locations errors in the QTDB (N/A: not applicable, N/R: not reported, TE: time error)

\begin{tabular}{|c|c|c|c|c|}
\hline Approach & Se (\%) & P+ $(\boldsymbol{\%})$ & $\begin{array}{c}\text { T-wave peak: } \\
\mathbf{T E}(\boldsymbol{m} \pm \boldsymbol{s} \boldsymbol{d})(\boldsymbol{m} \boldsymbol{s})\end{array}$ & $\begin{array}{c}\text { T-wave end: } \\
\mathbf{T E}(\boldsymbol{m} \pm \boldsymbol{s} \boldsymbol{d})(\boldsymbol{m} \boldsymbol{s})\end{array}$ \\
\hline Presented approach & 99.32 & 99.47 & $1.4 \pm 9.0$ & $2.8 \pm 15.3$ \\
\hline Arturo Martínez et al. $[17]$ & 99.20 & 99.01 & $5.3 \pm 12.9$ & $5.8 \pm 22.7$ \\
\hline Ghaffari et al. $[18]$ & 99.87 & 99.80 & $0.3 \pm 4.1$ & $0.8 \pm 10.7$ \\
\hline J. P. Martínez et al. $[15]$ & 99.77 & 97.79 & $0.2 \pm 13.9$ & $-1.6 \pm 18.1$ \\
\hline Vila et al. $[9]$ & 92.6 & N/R & $-12 \pm 23.4$ & $0.8 \pm 30.3$ \\
\hline Zhang et al. $[11]$ & N/R & N/R & N/A & $0.31 \pm 17.43$ \\
\hline
\end{tabular}

\section{Discussion}

Processing paced beats from ICM patients implies both an innovative and a challenging task, as we can find different kinds of restrictions and morphology changes which are not seen in normal sinus beats, such as considerable shortening of the ST-segment, wide QRS complexes, P-waves superimposing the ST-segment or even the T-wave waveform, alternation of specific morphologies, such as monophasic T-wave and biphasic T-wave in a single beat train, and so on. In addition, each lead represents the ventricular repolarisation activity with a specific morphology, time-duration and signal-to-noise ratio. According to the most recent available publications up to our knowledge, there is no other published work which has validated the T-wave peak and T-wave end locations using all the available 12 leads for paced ECG signals.

The approach is not time-consuming because the parameters of the model are defined in a training stage, which makes use of a number of the earliest intervals between each QRS offset and subsequent QRS onset. The structure of the training and analysis stages provides the system with robustness to a wide variety of T-wave morphologies and adaptability for eventual morphology changes, as well as for the incidence of physiological artefacts or baseline fluctuations. Even after the training stage had defined a kernel which best fits the predominant T-wave morphology, we can initiate other learning processes in the analysis stage if we identify significant morphology variations.

The overall results of T-wave detection rates shown in Table 2, concerning the database of 12-lead paced ECG signals, and Table 5, for QT database, indicate that the proposed kernel of skewed Gaussian function is suitable for modelling the T-wave with high accuracy for a wide variety of morphologies, as it is also observed through Figures 6(a)-6(q). These results in themselves indicate an improvement over the approaches reported by Arturo Martínez et al. [17] and Vila et al. [9].

Regarding the overall averages and standard deviations of the time errors related to T-wave peak and T-wave end locations presented in Table 3 and Table 5, for the used databases, we see that the method provides automatic locations with both high accuracy and repeatability. For the results presented in Table 6, both accuracy (less than one sampling period) and repeatability (between two and three sampling periods) are higher than those ones reported by Vila et al. [9] and Arturo Martinez et al. [17]. For T-wave end location, the repeatability is higher than four other approaches $[9,11,15,17]$. Considering also the inherent challenges related to paced beats and cardiac ischemic diseases, these results can be considered outstanding.

Concerning the results detailed in Table 4, the precordial leads V2-V4 produced the lowest timeerrors (lowest average and standard deviation values) for T-wave peak location and the precordial leads V1-V5 produced the most consistent results for T-wave end location. For the standard 
deviations (repeatability), minimum values of $1.12 \mathrm{~ms}$ (lead V3), for T-wave peak, and $9.45 \mathrm{~ms}$ (lead V5), for T-wave end, were obtained. The corresponding maximum values were, respectively, $18.65 \mathrm{~ms}$ and $33.30 \mathrm{~ms}$, both considering lead I, which is related to one of the lowest median SNR levels in the T-wave search windows.

\section{Conclusions}

This paper presents an innovative approach for automatic T-wave peak detection and T-wave end location based on a mathematical model of a skewed Gaussian function in 12-lead paced ECG signals. The results obtained suggest that the proposed kernel is able to model a wide diversity of T-wave morphologies (both paced and non-paced ones) and that it has a good performance in noisy conditions. Other important advantage is that the modelling of the T-wave does not depend on any empiric factor and neither the intervention of human experts. The kernel also adapts itself when morphology changes occur throughout a patient's signal.

The most significant contributions from this work were the proposal of an innovative and simple mathematical function to model the T-wave behaviour, the achievement of correct $\mathrm{T}$-wave detections (higher than 99\%) when applied over 12-lead paced ECG signals from patients with ischemic cardiomyopathy diseases and the publicly available records of the QT database, the accurate and consistent automatic locations of T-wave peak and T-wave end, and the comparison of the accuracy and repeatability of the automatic detections on all the standard twelve leads considering the SNR behaviour for each lead.

\section{Acknowledgements}

J.P.V. Madeiro wishes to thank CNPq, the Brazilian Research Council, for the Science Without Borders scholarship that allowed him to travel to the UK for part of his doctoral studies, the University Hospitals of Leicester NHS Trust, Leicester, UK, and the Laboratory of Bioengineering at the University of Leicester.

\section{Competing Interests}

None of the authors have any financial and personal relationships with other people or organisations that could inappropriately influence (bias) our work.

\section{Sources of funding}

Science Without Borders scholarship (process n. 202395/2011-1) from CNPq, the Brazilian Research Council, University of Leicester and Leicester NIHR Cardiovascular Biomedical Research Unit. 


\section{Ethical Approval}

Ethical approval was granted by the Derbyshire Research Ethics Committee (09/H0401/70) and the study protocol was approved by the Research and Development Office of the University Hospitals of Leicester National Health Service Trust (Leicester, UK). All patients gave written, informed consent.

\section{References}

[1] Madeiro JPV, Cortez PC, Marques JAL, Seisdedos CRV, Sobrinho CRMR. An innovative approach of QRS segmentation based on first-derivative, Hilbert and Wavelet Transforms. Medical Engineering \& Physics, vol. 34, pp. 1236-1246, 2012.

[2] Nicolson WB, McCann GP, Brown PD, Sandilands AJ, Stafford PJ, Schlindwein FS, Samani NJ, Ng GA. A Novel Surface Electrocardiogram Based Marker of Ventricular Arrhythmia Risk in Patients with Ischemic Cardiomyopathy. Journal of the American Heart Association, August 2012, vol. 1, issue 4, pp. 1-10, DOI - 10.1161/JAHA.112.001552.

[3] Hsing JM, Selzman KA, Leclercg C, Pires LA, McLaughlin MG, McRae SE, Peterson BJ, Zimetbaum PJ. Paced left ventricular QRS width and ECG parameters predict outcomes after cardiac resynchronization therapy: PROSPECT-ECG substudy. Circulation: Arrhythmia and Electrophysiology, vol. 4, n. 6, pp. 851-857, 2011.

[4] Vázquez-Seisdedos CR, Neto JE, Reyes EJM, Klautau A, Oliveira RCL. New approach for Twave end detection on electrocardiogram: Performance in noisy conditions. BioMedical Engineering Online, vol. 10, n. 77, pp. 1-11, 2011.

[5] Bloomfield DM, Steinman RC, Namerow PB, Parides M, Davidenko J, Kaufman ES, Shinn T, Curtis A, Fontaine J, Holmes D, Russo A, Tang C, Bigger JT. Microvolt T-Wave Alternans Distinguishes Between Patients Likely and Patients Not Likely to Benefit From Implanted Cardiac Defibrillator Therapy: A Solution to the Multicenter Automatic Defibrillator Trial (MADIT) II Conundrum. Circulation, vol. 110, pp. 1885-1889, 2004.

[6] van der Avoort CJ, Filion KB, Dendukuri N, Brophy JM. Microvolt T-wave alternans as a predictor of mortality and severe arrhythmias in patients with left-ventricular dysfunction: $a$ systematic review and meta-analysis. BMC Cardiovascular Disorders, vol. 9, n. 5, pp. 1-9, 2009.

[7] Lee KW, Okin PM, Kligfield P, Stein KM, Lerman BB. Precordial QT dispersion and inducible ventricular tachycardia. American Heart Journal, vol. 134, n. 6, pp. 1005-1013, 1997.

[8] Oikarinen L, Nieminen MS, Viitasalo M, Toivonen L, Jern S, Dahlöf B, Devereux RB and Okin PM. QRS Duration and QT Interval Predict Mortality in Hypertensive Patients with Left Ventricular Hypertrophy: The Losartan Intervention for Endpoint Reduction in Hypertension Study. Hypertension, vol. 43, pp. 1029-1034, 2004.

[9] Vila JA, Gang Y, Presedo JMR, Delgado MF, Barro S, Malik M. A New Approach for TU Complex Characterization. IEEE Transactions on Biomedical Engineering, vol. 47, n. 6, pp. 764-772, 2000. 
[10] Murray A, McLaughlin NB, Bourke JP, Doig JC, Furniss SS, Campbell RW. Errors in manual measurement of QT intervals. British Heart Journal, vol. 71, pp. 386-390, 1994.

[11] Zhang Q, Manriquez AI, Médigue C, Papelier Y, Sorine M. An Algorithm for Robust and Efficient Location of T-wave Ends in Electrocardiograms. IEEE Transactions on Biomedical Engineering, vol. 53, n. 12, pp. 2544-2552, 2006.

[12] Yana K, Shichiku H, Satoh T, Mizuta H, Ono T. An Improved QT Interval Measurement based on Singular Value Decomposition. Proceedings of the 28th IEEE EMBS Annual International Conference. New York City, USA, 2006.

[13] Zong W, Saeed M, Heldt T. A QT Interval Detection Algorithm Based on ECG Curve Length Transform. Computers in Cardiology, vol. 33, pp. 377-380, 2006.

[14] Meste O, Janusek D, Kania M, Maniewski R. T Waves Segmentation and Analysis Using Inverse Normalized Integrals. 33rd Annual International Conference of the IEEE EMBS. Boston, Massachusetts, USA, 2011.

[15] Martínez JP, Almeida R, Olmos S, Rocha AP, Laguna P. A wavelet-based ECG delineator: Evaluation on standard databases. IEEE Transactions on Biomedical Engineering, vol. 51, n. 4, pp. 570-581, 2004.

[16] Madeiro JPV, Cortez PC, OliveiraFI, Siqueira RS. A new approach to QRS segmentation based on wavelet bases and adaptive threshold technique. Medical Engineering and Physics, vol. 29, pp. 26-37, 2007.

[17] Martínez A, Alcaraz R, Rieta JJ. Application of the phasor transform for automatic delineation of single-lead ECG fiducial points. Physiological Measurement, vol. 31, pp. 1467-1485, 2010.

[18] Ghaffari A, Homaeinezhad MR, Akraminia M, Atarod M, Daevaeiha M. A robust waveletbased multi-lead electrocardiogram delineation algorithm. Medical Engineering and Physics, vol. 31, pp. 1219-1227, 2009.

[19] Almeida R, Martínez JP, Rocha AP, Laguna P. Multilead ECG delineation using spatially projected leads from Wavelet Transform loops. IEEE Transactions on Biomedical Engineering, vol. 56, n. 8, pp. 1996-2005, 2009.

[20] Christov I, Simova I. Q-onset and T-end delineation: assessment of the performance of an automated method with the use of a reference database. Physiological Measurement, vol. 28, pp. 213221, 2007.

[21] The CSE Working Party: Recommendations for measurement standards in quantitative electrocardiography. European Heart Journal, vol. 6, pp. 815-825, 1985.

[22] Last T, Nugent CD, Owens FJ. Multi-component based cross-correlation beat detection in electrocardiogram analysis. BioMedical Engineering Online, vol. 03, n. 26, pp. 1-14, 2004.

[23] J. Richardson, L. J. Haywood, V. K. Murthy and G. Harvey. A mathematical model for ECG wave forms and power spectra. Mathematical Biosciences, vol. 12, pp. 321-328, 1971. 
[24] B. Wohlfart. A simple model for demonstration of STT-changes in ECG. Eur. Heart J., vol. 8, pp. 409-416, 1987.

[25] M. Malik and A. J. Camm. Computer model of cardiac repolarization processes and of the recovery sequence. Comput. Biomed. Res., vol. 22, pp. 160-180, 1989.

[26] R. Padrini, G. Butrous, A. J. Camm, and M. Malik. Algebraic decomposition of the TU wave morphology patterns. PACE, vol. 18, pp. 2209-2215, 1995.

[27] Laguna P, Mark R, Goldberger A, Moody GB. A database for evaluation of algorithms for measurement of QT and other waveform intervals in the ECG. Computers in Cardiology, vol 24, pp. 295-298, 1997.

[28] Ibañez A. The ECG T-wave duration as an index of dispersion of ventricular repolarization: insights from simulations. Computers in Cardiology, vol. 33, pp. 793-796, 2006.

[29] Arini P, Valverde E, Beltran G, Laguna P. Quantification of ventricular repolarization dispersion on the electrocardiogram by means of $T$ wave duration. Computers in Cardiology, vol. 31, pp. 757-760, 2004.

[30] Mincholé A, Martínez JP, Arini P, Risk M, Laguna P. T wave width alterations during valsalva maneuver in diabetic patients. Computers in Cardiology, vol. 33, pp. 709-712, 2006. 